\title{
Surfactant Protein A Expression in Chronic Rhinosinusitis and Atrophic Rhinitis
}

\author{
Mohammad Waheed El-Anwar ${ }^{1}$ Atef A. Hamed ${ }^{1}$ Abd ElRaof Said Mohamed ${ }^{1}$ \\ Ahmad Abdel-Fattah Nofal ${ }^{1}$ Maha A. Mohamed ${ }^{2}$ Hesham R. Abdel-Aziz ${ }^{3}$ \\ ${ }^{1}$ Department of Otorhinolaryngology, Head and Neck Surgery, \\ Zagazig University, Zagazig, Egypt \\ 2 Department of Clinical Pathology, Zagazig University, Zagazig, Egypt \\ Address for correspondence Mohammad El-Anwar, MD, Department \\ of Otorhinolaryngology Head and Neck Surgery, Zagazig University, \\ Zagazig 0020552309843, Egypt (e-mail: mwenteg@yahoo.com).
}

${ }^{3}$ Department of Pathology, Zagazig University, Zagazig, Egypt

Int Arch Otorhinolaryngol 2015;19:130-134.

\begin{abstract}
Introduction Surfactant protein A (SP-A) exhibits antimicrobial properties and interacts with a variety of respiratory tract pathogens.

Objective The objective of this study was to detect the presence of SP-A and measure its alterations in chronic rhinosinusitis (CRS) and primary atrophic rhinitis (PAR) versus healthy controls.

Methods Inferior turbinate and sinus mucosal biopsies were taken from 30 patients with CRS, 30 patients with PAR, and 20 healthy controls. Immunohistochemical staining for SP-A and polymerase chain reaction (PCR) amplification of SP-A messenger RNA were performed on nasal tissue samples.

Results Immunostaining localized SP-A to the mucosa and submucosal glands in CRS specimens but failed to localize it in PAR specimens. Quantitative PCR showed a high, statistically significant increase in the SP-A levels of patients with CRS when compared with controls $(p<0.0001)$ and also demonstrated a significant reduction of SP-A in patients with PAR compared with controls $(p<0.005)$.

Keywords

- sinusitis

- rhinitis

- nose

Conclusion SP-A is significantly increased in CRS and decreased significantly in PAR and appears to be expressed by respiratory epithelial cells and submucosal glandular elements of the sinonasal mucosa. The potential therapeutic applications of surfactant in the enhancement of mucociliary clearance need to be studied.
\end{abstract}

\section{Introduction}

Pulmonary surfactant is secreted as lamellar bodies from type II pneumocytes to reduce surface tension at the air-liquid interface. Over $90 \%$ of pulmonary surfactant is lipid, whereas the remainder is composed of plasma proteins and the surfactant proteins (SPs) A, B, C, and D. SPs are hydrophilic proteins actively involved in the innate immune response to inhaled pathogens. SPs are part of the collectin family of proteins secreted at several epithelial surfaces in the body. ${ }^{1}$

SP-A, hydrophilic sialoglycoprotein, exhibits antimicrobial properties and interacts with a variety of respiratory tract pathogens that have related molecular structures, called pathogen-associated molecular patterns (PAMP). PAMPs differ little from one pathogen to another but are not found in the host. ${ }^{2}$ SP-A bind PAMPs located on microbial membranes via their calcium-dependent carbohydrate-binding domains. Independent of direct binding to pathogens, SP-A also interacts directly with dendritic cells, modulates subsequent $\mathrm{T}$-cell responses, optimizes leukocyte function and chemotaxis, modifies subsequent cytokine/chemokine profiles, and activates complement. Thus, these proteins are crucial in the initial interaction, recognition, processing, and subsequent adaptive immune received

November 23, 2014

accepted

January 10, 2015

published online

February 20, 2015
DOI http://dx.doi.org/

$10.1055 / \mathrm{s}-0035-1546432$. ISSN $1809-9777$.
Copyright $(2015$ by Thieme Publicações License terms

Ltda, Rio de Janeiro, Brazil

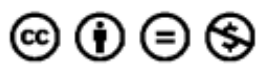


response for a wide variety of inhaled pathogens and allergens. ${ }^{3}$ Human SP-A is encoded by two genes, SP-A1 and SP-A2. ${ }^{2}$

Bronchoalveolar lavage specimens from patients with chronic bronchial asthma or pneumocystis pneumonia have demonstrated increased SP-A. ${ }^{3}$ SP-A expression has also been assessed in the sinonasal mucosa. In a semiquantitative study, Dutton et al found elevated SP-A in the mucosa of rabbits with intercurrent sinusitis or antibiotic-treated sinusitis compared with pathogen-free animals. ${ }^{4}$

Diseases of the sinonasal system, specifically chronic rhinosinusitis (CRS), are often associated with pulmonary system diseases. Asthma sensitive to the ingestion of aspirin may be associated with nasal polyps. Allergic fungal rhinosinusitis in the upper airway could be associated with allergic bronchopulmonary aspergillosis. ${ }^{5}$ Patients with cystic fibrosis invariably develop CRS in addition to their debilitating pulmonary disease. These are attributed to similar mechanisms of inspissated mucus, impaired mucociliary clearance, and persistent bacterial infections and inflammation. Moreover, the lungs and the paranasal sinuses share contact with inhaled pathogens and include many of the same epithelial properties. ${ }^{6}$

SPs appear to have an important function in immunologic and rheological process of the nasal mucosa and support the therapeutic use of liposomal nasal sprays. ${ }^{7}$

The aim of this study was to investigate whether SP-A is present in sinonasal mucosa and compare expression in distinct types of chronic rhinitis [CRS and primary atrophic rhinitis (PAR)] versus healthy control mucosa.

\section{Materials and Methods}

This study was performed from February 2011 to September 2014. Informed written consent was obtained from included subjects, and the Institutional Review Board approved the study. The subjects were divided into three groups; the first group included 30 patients with persistent bacterial CRS despite appropriate medical treatment and scheduled for endoscopic sinus surgery. Diagnosis of CRS depended on Meltzer et al criteria of symptoms that persist more than 12 weeks. Sinus mucosa was biopsied during the course of endoscopic sinus surgery. ${ }^{8}$

The second group included 30 patients with PAR randomly selected from the outpatient clinic. Diagnosis of atrophic rhinitis followed Ly et al diagnostic criteria for atrophic rhinitis with two or more clinical features such as recurrent epistaxis, episodic anosmia, physician-documented nasal purulence, or nasal crusting for $\geq 6$ months with atrophied inferior turbinate with no detected cause. ${ }^{9}$ Cases known to be secondary atrophic rhinitis and patients with history of nasal trauma, sinonasal surgery, granulomatous disease, or radiation to the sinonasal area were excluded. Nasal mucosal biopsies were taken from inferior turbinate under local anesthesia.

The third group included 20 normal controls; nasal mucosal biopsies were obtained from these subjects undergoing surgery for other reasons other than CRS such as rhinoplasty (10 cases from normal inferior turbinate mucosa) and zygo- maticomaxillary fractures (10 cases from normal maxillary sinus mucosa).

Immunohistochemical staining for SP-A and polymerase chain reaction (PCR) amplification of SP-A messenger RNA were performed on resected nasal tissues.

\section{Immunohistochemical Analysis}

Biopsy specimens were washed three times with iced phosphate-buffered saline for 5 minutes to remove any exogenous SP-A that may have migrated from the lower airways. After thawing to $-20^{\circ} \mathrm{C}$ and overnight decalcification, followed by fixation in $4 \%$ paraformaldehyde, 4 - $\mu$ m-thick sections were cut from paraffin blocks containing representative tissue samples. Paraffin sections were dewaxed in xylene, rehydrated through a graded alcohol series, placed in $10 \mathrm{mmol} / \mathrm{L}$ of citrate buffer, and submitted to heat retrieval using a vapor lock for 40 minutes. After heating, the slides were allowed to cool to room temperature and briefly washed with Tris (hydroxymethyl) amino methane-buffered saline. Endogenous peroxidase activity was blocked with $3 \%$ hydrogen peroxide in methanol for 5 minutes. Normal serum (Novo stain Super ABC kit, Novocastra, Newcastle upon Tyne, England) was used for 30 minutes to block nonspecific immunostaining. Immunohistochemical staining was performed using a standard avidin-biotin peroxidase system (Novo stain Super ABC kit). The monoclonal antibody SP-A (isotype; immunoglobulin [Ig] G2a purchased from Abcam) were used in concentration of 1/ 200. Two slides for SP-A antibodies and isotypic control (mouse monoclonal IgG2a as negative control) were incubated overnight at room temperature. Following washing in phosphatebuffered saline, biotinylated universal secondary antibody (Novo stain Super ABC kit) was applied for 30 minutes. The sections then were incubated with the avidin-biotin complex reagent (Novo stain Super ABC kit) for 30 minutes and developed with 3, 3-diaminobenzidine tetrahydrochloride in phosphate-buffered saline ( $\mathrm{pH} 7.5$ ) containing $0.036 \%$ hydrogen peroxide for 5 minutes. Light Mayer hematoxylin was applied as a counterstain. The slides then were dehydrated in a series of ethanol and mounted with permount. Cytoplasmic or membranous staining was considered as positive. Two negative control sections were used in each case; one was incubated with the secondary antibody only, the other with the primary antibody only. Sections of human lung stained with anti SP-A antibodies were used for positive control. Cells were considered positive for anti SP-A antibodies when at least $10 \%$ of cells show diffuse cytoplasmic or membranous staining.

\section{Quantitative Reverse-Transcriptase Polymerase Chain Reaction}

Total RNA purification was performed on all specimens (using an Aurum Total RNA Mini Prep-Kit; Bio-Rad, Hercules, California, United States), including a DNase 1 digest, according to the manufacturer's instructions. Reverse transcriptase PCR was performed on a portion of the purified RNA for each specimen to create complementary DNA (cDNA) libraries using the iScript cDNA Synthesis Kit (Bio-Rad) under the following conditions in a conventional thermocycler: 5 minutes at $25^{\circ} \mathrm{C}, 30$ minutes at $42^{\circ}$ $\mathrm{C}$, and 5 minutes at $85^{\circ} \mathrm{C}$. 


\section{Quantitative Polymerase Chain Reaction}

Intron-exon spanning SP-A primers were used in the quantitative PCR amplification reactions with the following design: 5'-AAGCCACACTCCACGACTTTAGA-3' and 5'-GCCCATTGCTGGAGAAGACCT- 3' (Integrated DNA Technology Inc., Coralville, Iowa, United States). Commercially available _-actin primers for the amplification of the housekeeping gene were used: 5-TCATGAAGTGTGACGTTGACATCCGT-3 and 5'-CTTAGAAGCATTTGCGGTGCACGATG-3 (Promega, Madison, Wisconsin, United States). In a quantitative PCR detection system (My iQ Thermal Cycler; Bio-Rad), 96-well reaction plates were used to perform real-time, quantitative PCR with the sample wells containing the following: $25 \mu \mathrm{L} 2$ _ Supermix with SYBR green (a fluorescent double-stranded DNA-binding reporter molecule), 1 $\mu \mathrm{L}$ each of SP-A primer $(100 \mathrm{ng} / \mu \mathrm{L})$ or_-actin primers ( $100 \mathrm{pmol} /$ $\mu \mathrm{L})$ for control wells, a $1-\mu \mathrm{L}$ sample complementary DNA, and 22 $\mu \mathrm{L}$ of nuclease-free water. After an initial activation step at $95^{\circ} \mathrm{C}$ for 3 minutes, amplification was performed across 45 cycles with the following parameters: denaturation for 30 seconds at $95^{\circ} \mathrm{C}$, annealing for 20 seconds at $58^{\circ} \mathrm{C}$, and elongation for 30 seconds at $72^{\circ} \mathrm{C}$. Digital capture of SYBR green fluorescence was performed during the annealing phase for threshold cycle assessment. The threshold cycle values were automatically stored in an output file, and these were analyzed to determine relative starting messenger RNA (mRNA) concentrations according to the 2-_threshold cycle method16 using a proprietary macro (Bio-Rad) with Excel 2003 XT (Microsoft Corp., Redmond, Washington, United States). A melt-curve analysis confirmed specimen uniformity and estimated product size in each sample well in the 96-well plate. The following protocol was programmed into the My iQ Thermal Cycler (Bio-Rad) to produce the melt curves for each sample well after amplification using SYBR green as the reporter: 1 minute at $95^{\circ} \mathrm{C}, 1$ minute at $55^{\circ} \mathrm{C}$, and 10 seconds at $55^{\circ} \mathrm{C}$ for 80 cycles. Finally, to verify PCR product sequence, random samples were prepared and sequenced by the Vanderbilt University (Nashville, Tennessee, United States) DNA sequencing core laboratory. Data were compared with the human genome via BLAST downloadable software (http:// www.ncbi.nlm.nih.gov/genome/seq/BlastGen/BlastGen.cgi? taxid=9606).

\section{Statistical Analysis}

Statistical analyses were performed using SPSS 14.0 statistical software for Windows (SPSS Inc., Chicago, Illinois, United States). The significance level was set at $p<0.05$. Quantitative data (age and quantitative PCR) in patient and control groups were registered and compared using $t$ test, and sex differences between the three groups were analyzed by chi-square test.

\section{Results}

Eighty subjects were included in current study: 30 patients with CRS, 30 with PAR, and 20 controls. The mean age ( \pm standard deviation) was $37( \pm 14.2)$ years. The three groups were matched for $\operatorname{sex}(p=0.84$, chi-square $=0.341)$ and sex as nonsignificant differences in age between CRS and controls ( $p=0.64, t=0.47$ ), and nonsignificant differences between PAR and controls $(p=0.97, t=0.035)$ were reported (-Table $\mathbf{1}$ ). There was strong positive staining in the nasal mucosa and submucosa in CRS, as shown in - Fig. 1. This indicates that SP-A is secreted by nasal epithelium and glandular epithelium. Faint staining for SP-A was detected in biopsies of control specimens as shown in -Fig. 2, and staining for SP-A was not detected in biopsies of patients with atrophic rhinitis (-Fig. 3). As a negative staining control, sections were incubated with nonimmune rabbit serum instead of SP-A antibody, resulting in loss of staining.

Quantitative PCR (reported as $\triangle \mathrm{CT} \pm$ standard deviation) showed highly significantly increased levels of SP-A $(4.69 \pm 2.7)$ mRNA $(t=6.6417, p<0.0001)$ from patients with CRS when compared with controls (10.4 \pm 3.15$)$. Although SP-A mRNA was decreased in patients with PAR $(15.6 \pm 6.25)$ when compared with controls $(10.4 \pm 3.15)$, this decrease was statistically significant $(t=3.8778, p=0.0003)$.

\section{Discussion}

SP-A plays an integral part in the innate defense system. SP-A expression and function are altered in a variety of inflammatory and infectious diseases. However, the presence of SP-A in the human paranasal sinus mucosa is not well known. ${ }^{7}$

There are few previous studies investigating surfactant and SP in the upper airway. ${ }^{10}$ The presence of lamellar bodies and SP-A, -B, and -D have been described in the porcine eustachian tube, indicating that a surfactant-like system is present outside the pulmonary system. ${ }^{11}$ Woodworth et al demonstrated the presence of lamellar bodies, SP, and mRNA in both diseased and normal human sinus tissue and reported that SP-A and SP-D immunolocalized to the sinonasal epithelium and submucosal gland secretory ducts. ${ }^{12}$ The discovery

Table 1 Age and sex of the patients (CRS, PAR) and control (Healthy) groups

\begin{tabular}{|c|c|c|c|c|c|c|c|}
\hline \multirow[t]{2}{*}{ Subjects } & \multicolumn{2}{|c|}{ CRS } & \multicolumn{2}{|c|}{ PAR } & \multicolumn{2}{|c|}{ Controls } & \multirow[t]{2}{*}{$P$ value } \\
\hline & $n$ & $\%$ & $n$ & $\%$ & $n$ & $\%$ & \\
\hline \multicolumn{8}{|l|}{ Sex } \\
\hline Male & 18 & 60 & 16 & 53.3 & \multirow{2}{*}{$\begin{array}{l}12 \\
8\end{array}$} & \multirow{2}{*}{$\begin{array}{l}60 \\
40\end{array}$} & \multirow{2}{*}{$\begin{array}{l}0.84 a \\
\text { NS }\end{array}$} \\
\hline Female & 12 & 40 & 14 & 46.7 & & & \\
\hline Age & \multicolumn{2}{|c|}{$29.5 \pm 10.1$} & \multicolumn{2}{|c|}{$28.3 \pm 9.7$} & \multicolumn{2}{|c|}{$29.6 \pm 9.6$} & $N S^{b}$ \\
\hline
\end{tabular}

Abbreviations: CRS, chronic rhinosinusitis; PAR, primary atrophic rhinitis; NS, not significant.

${ }^{a}$ Chi-square test.

${ }^{\mathrm{b}}$ Differences between CRS and healthy $(p=0.64, t=0.47)$ and differences between PAR and healthy $(p=0.97, t=0.035)$. 

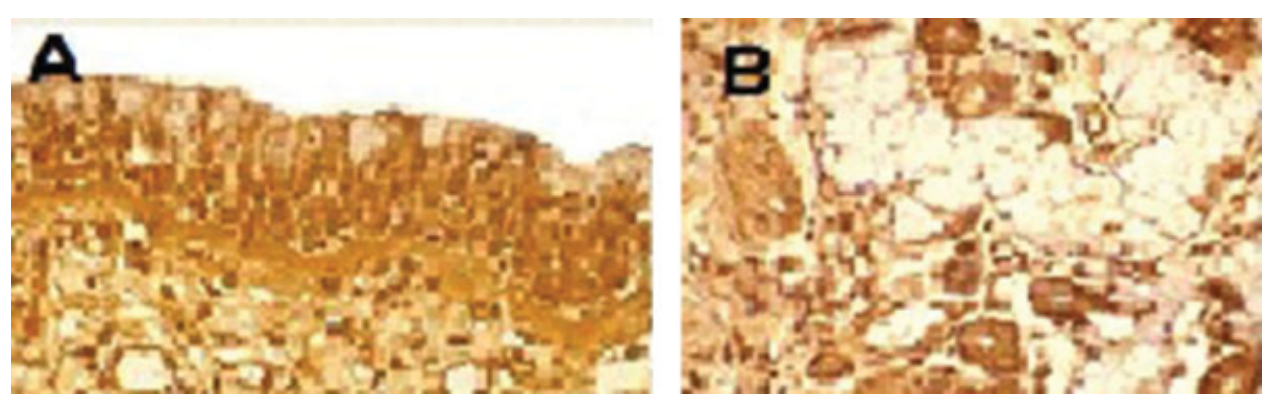

Fig. 1 Photomicrograph of chronic rhinosinusitis showing strong cytoplasmic immunostaining of surfactant protein-A in both nasal mucosa (A) and submucosal glands (B). Hematoxylin counterstain $\times 400$.
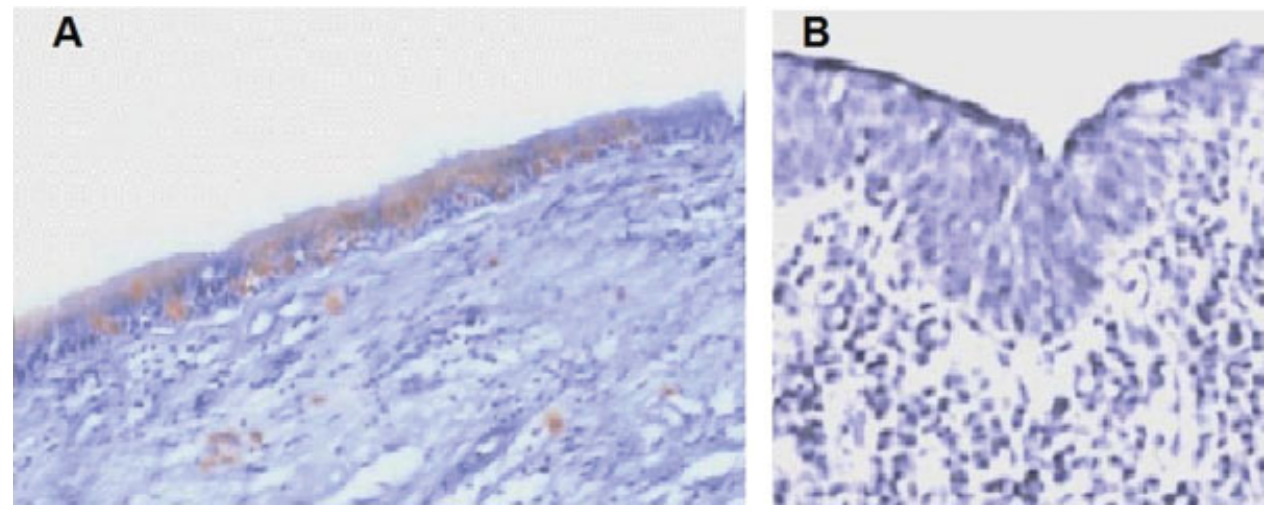

Fig. 2 (A) Photomicrograph showing faint immunostaining of surfactant protein A (SP-A) in human nasal mucosa and submucosa of the control group; hematoxylin counterstain $\times 400$. (B) Photomicrograph of atrophic rhinitis showing negative immunostaining for surfactant protein-A (SP-A) in human nasal mucosa and submucosa; hematoxylin.
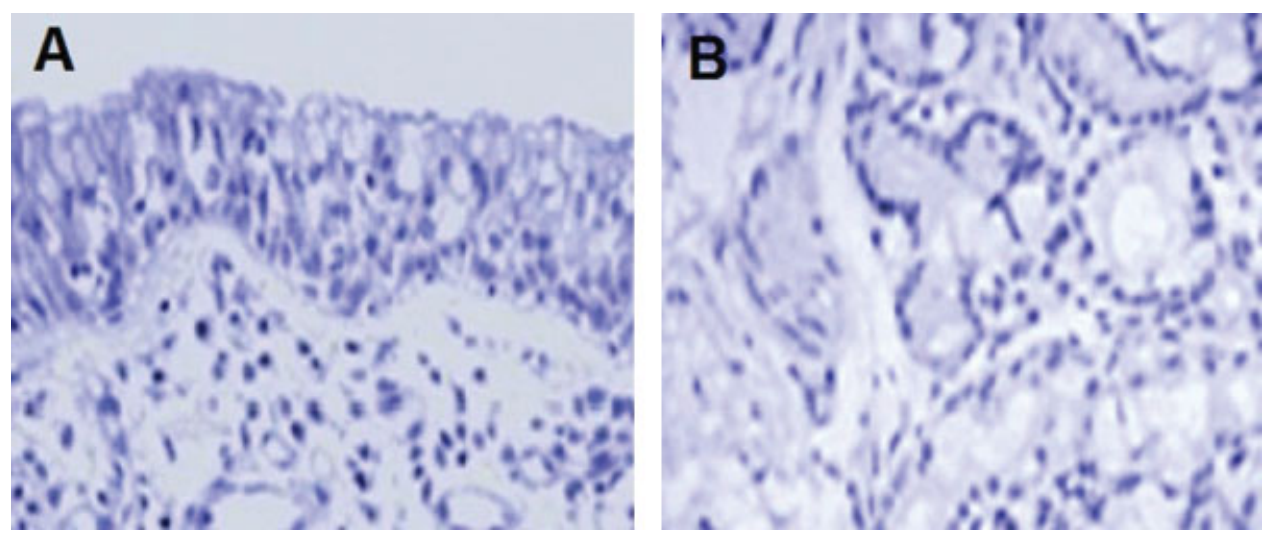

Fig. 3 Photomicrograph showing negative control with negative immunostaining for surfactant protein-A (SP-A) in nasal mucosa (A) and submucosa (B). Hematoxylin counterstain $\times 400$.

of surfactant production and secretion in sinonasal mucosa indicates that initial contact and interaction between pathogens and SPs occurs after inhalation and deposition into the mucus of the upper respiratory tract.

This study characterizes the differential expression of SP-A in the nasal mucosal samples of patients with CRS using immunohistochemical staining and quantitative PCR. By immunohistochemistry, we detected strong SP-A staining in the epithelial cells and lumen of the submucosal glands in the CRS patients. Also SP-A mRNA increased significantly in CRS patients and decreased significantly in patients with PAR.
Strong SP-A staining in the epithelial cells and lumen of the submucosal glands in the CRS could be explained on the basis of the different histologic findings present in normal mucosa and CRS mucosa, because SP production mostly occurs in submucosal glandular pathways and these patients exhibit glandular hyperplasia. This hyperplasia is more pronounced in patients with CRS. This may have contributed more mRNA transcripts due to a relative abundance of these cell types. In agreement, Woodworth et al reported a significant elevation of SP-A and -D in patients with CRS associated with cystic fibrosis. ${ }^{6}$ In the sinonasal tissue of rabbits with acute bacterial 
sinusitis (inflammation but not allergy), SP-A was more prevalent than in pathogen-free animals. ${ }^{3}$

The SP-A protein levels were significantly reduced compared with controls in PAR patients. A possible explanation is that absence of significant SP-A gene up-regulation and depletion of SP-A protein reserves in the presence of atrophic changes of the submucosal glands and mucosa. This is in agreement with the results obtained by Sayed et al, who had demonstrated a significant decrease in phospholipid and surfactant concentrations in the cases with PAR compared with normal cases. ${ }^{13}$

Several theories tried to explain the etiology of PAR. Infection, one of the theories, can affect the surfactant system leading to surfactant deficiency through several mechanisms including inflammatory cytokines produced in response to sepsis inhibiting the synthesis of surfactant. ${ }^{14}$ Activated neutrophils are capable of cleaving SP-A and impairing surfactant function. ${ }^{15}$ Sayed et al reported that abnormal surfactant function in the larger airways might be expected to decrease the efficiency of mucociliary clearance and also adversely lead to stasis of mucus. ${ }^{13}$

In addition, surfactant deficiency will impair opsonization and phagocytosis of bacteria by macrophages, thus favoring bacterial multiplication and leukocyte infiltration. Due to a lack of the opsonizing effect (due to decreased concentration of secretory IgA and surfactant deficiency), the leukocytes cannot phagocytose and destroy the bacteria with continuous production of proteolytic enzymes in the secretion, which are probably harmful to the mucous membrane, as it is known that inhalation of proteolytic enzymes produces emphysema in animals. Our results support the previously reported concept that SP has an important function in immunologic process of the nasal mucosa. $^{7}$

The therapeutic potential of correcting abnormalities in SPs has already been demonstrated in the lower airways. The correction of SP-D deficiencies with topical SP-D reverses emphysema, pulmonary lipidosis, and macrophage infiltrations in diseased mice. ${ }^{15}$ Gesche et al found that human (rh) KGF, betamethasone, or their combination treatment increased secreted surfactant phosphatidylcholines in neonatal rats with lung injuries. ${ }^{16}$ Furthermore, topical SP-A and SP-D decreased IgE and eosinophilia in mouse models of allergic bronchopulmonary aspergillosis. ${ }^{17}$ Because allergic bronchopulmonary aspergillosis is very similar to allergic fungal rhinosinusitis and can be considered its counterpart in the lower airway, this could lead to future therapeutic options for difficult to treat sinus disease, as Tan et al found that the surfactant does not appear to elicit cellular toxicity using an in vitro explant model. ${ }^{18}$

\section{Conclusion}

This study characterized the detection and expression of SP-A in human sinus mucosa. SP-A is significantly increased in CRS and decreased in PAR and appears to be expressed by respiratory epithelial cells and submucosal glandular elements of the sinonasal mucosa. Further characterization of this specialized protein is warranted considering the poten- tial therapeutic applications of surfactant in the enhancement of mucociliary clearance.

\section{References}

1 Woodworth BA, Smythe N, Spicer SS, Schulte BA, Schlosser RJ. Presence of surfactant lamellar bodies in normal and diseased sinus mucosa. ORL J Otorhinolaryngol Relat Spec 2005;67(4):199-202

2 Khubchandani KR, Snyder JM. Surfactant protein A (SP-A): the alveolus and beyond. FASEB J 2001;15(1):59-69

3 Wootten CT, Labadie RF, Chen A, Lane KF. Differential expression of surfactant protein $A$ in the nasal mucosa of patients with allergy symptoms. Arch Otolaryngol Head Neck Surg 2006;132(9): 1001-1007

4 Dutton JM, Goss K, Khubchandani KR, Shah CD, Smith RJH, Snyder JM. Surfactant protein A in rabbit sinus and middle ear mucosa. Ann Otol Rhinol Laryngol 1999;108(10):915-924

5 Shah A, Panjabi C. Allergic aspergillosis of the respiratory tract. Eur Respir Rev 2014;23(131):8-29

6 Woodworth BA, Wood R, Bhargave G, Cohen NA, Baatz JE, Schlosser RJ. Surfactant protein B detection and gene expression in chronic rhinosinusitis. Laryngoscope 2007;117(7):1296-1301

7 Schicht M, Knipping S, Hirt R, Beileke S, Sel S, Paulsen F, Bräuer L. Detection of surfactant proteins A, B, C, and D in human nasal mucosa and their regulation in chronic rhinosinusitis with polyps. Am J Rhinol Allergy 2013;27(1):24-29

8 Meltzer EO, Hamilos DL, Hadley JA, et al; American Academy of Allergy, Asthma and Immunology (AAAAI); American Academy of Otolaryngic Allergy (AAOA); American Academy of Otolaryngology-Head and Neck Surgery (AAO-HNS); American College of Allergy, Asthma and Immunology (ACAAI); American Rhinologic Society (ARS). Rhinosinusitis: establishing definitions for clinical research and patient care. J Allergy Clin Immunol 2004;114(6, Suppl):155-212

9 Ly TH, deShazo RD, Olivier J, Stringer SP, Daley W, Stodard CM. Diagnostic criteria for atrophic rhinosinusitis. Am J Med 2009; 122(8):747-753

10 Ooi EH, Wormald PJ, Carney AS, James CL, Tan LW. Surfactant protein $\mathrm{D}$ expression in chronic rhinosinusitis patients and immune responses in vitro to Aspergillus and alternaria in a nasal explant model. Laryngoscope 2007;117(1):51-57

11 Paananen R, Glumoff V, Sormunen R, Voorhout W, Hallman M. Expression and localization of lung surfactant protein $B$ in eustachian tube epithelium. Am J Physiol Lung Cell Mol Physiol 2001; 280(2):L214-L220

12 Woodworth BA, Neal JG, Newton D, et al. Surfactant protein A and $D$ in human sinus mucosa: a preliminary report. ORL J Otorhinolaryngol Relat Spec 2007;69(1):57-60

13 Sayed RH, Abou-Elhamd KE, Abdel-Kader M, Saleem TH. Study of surfactant level in cases of primary atrophic rhinitis. J Laryngol Otol 2000;114(4):254-259

14 Arias-Díaz J, Vara E, García C, Gómez M, Balibrea JL. Tumour necrosis factor-alpha inhibits synthesis of surfactant by isolated human type II pneumocytes. Eur J Surg 1993;159(10):541-549

15 Zhang L, Ikegami M, Dey CR, Korfhagen TR, Whitsett JA. Reversibility of pulmonary abnormalities by conditional replacement of surfactant protein D (SP-D) in vivo. J Biol Chem 2002;277(41):38709-38713

16 Gesche J, Fehrenbach H, Koslowski R, et al. rhKGF stimulates lung surfactant production in neonatal rats in vivo. Pediatr Pulmonol 2011;46(9):882-895

17 Kishor U, Madan T, Sarma PU, Singh M, Urban BC, Reid KB. Protective roles of pulmonary surfactant proteins, SP-A and SP-D, against lung allergy and infection caused by Aspergillus fumigatus. Immunobiology 2002;205(4-5):610-618

18 Tan NC, Cooksley CM, Paramasivan S, Vreugde S, Wormald PJ. Safety evaluation of a sinus surfactant in an explant-based cytotoxicity assay. Laryngoscope 2014;124(2):369-372 